\title{
Algorithm-Based Enrollment
}

National Cancer Institute

\section{Source}

National Cancer Institute. Algorithm-Based Enrollment. NCI Thesaurus. Code C154533.

An assertion of complete data capture between the start and end dates, based on a

locally developed or applied algorithm, often using multiple criteria. 